\title{
La discontinuación de dosis bajas de aspirina se asoció a una mayor incidencia de eventos cardiovasculares
}

Low-dose aspirin discontinuation was associated with a higher incidence of cardiovascular events

Sundström J y col. Circulation. 2017;136(13):1183-92.

\section{Objetivos}

Evaluar si la discontinuación de la aspirina recibida a largo plazo como prevención primaria o secundaria y a dosis bajas, provoca aumento de riesgo de padecer eventos cardiovasculares (CV).

\section{Diseño, lugar y población}

Estudio de cohorte de base poblacional que incluyó toda la población de Suecia expuesta a la aspirina (registro unificado de consumo de aspirina y venta bajo prescripción médica). Se incluyeron en el estudio 601.527 personas que entre 2005 y 2009 tenían más de 40 años de edad, no tenían antecedentes personales de cáncer y tenían una adherencia al tratamiento con aspirina de al menos el $80 \%$ durante el primer año observado.

\section{Medición de Resultados Principales}

El resultado principal fue la incidencia de eventos $\mathrm{CV}$, los cuales fueron identificados en los registros suecos de pacientes internados y de causas de muerte. Los primeros 3 meses después de un sangrado importante o un procedimiento quirúrgico fueron excluidos del análisis. Se realizó ajuste por edad, sexo, enfermedad CV previa, diabetes mellitus, medicamentos antiplaquetarios o anticoagulantes orales y por fármacos antinflamatorios esteroideos y no esteroideos orales.

\section{Resultados Principales}

Durante tres años de seguimiento (1.491.369 personas-año), ocurrieron 62.690 eventos CV, equivalente a una densidad de incidencia de 42 por 1000 personas-año. Un total de 73.636 personas murieron durante este tiempo. Fueron excluidos de los análisis 19.978 persona-año debido a procedimientos quirúrgicos y hemorragia mayor. La proporción acumulada de interrupción de la aspirina fue de $15 \%$ a los tres años. La mayoría de los participantes del estudio (54\%) utilizaban aspirina para prevención secundaria. Los pacientes que suspendieron la aspirina tuvieron un evento $\mathrm{CV}$ adicional por año cada 74 pacientes que la discontinuaron. En la Tabla 1 se pueden observar la densidad de incidencia y el riesgo de eventos CV según la exposición a aspirina y tipo de prevención.

Tabla 1: Riesgo de eventos cardiovasculares al discontinuar la aspirina

\begin{tabular}{|c|c|c|c|}
\hline Prevención & Aspirina & $\begin{array}{c}\text { Eventos CV cada } 1000 \\
\text { personas-año }\end{array}$ & $\begin{array}{l}\text { Hazard Ratio } \\
\text { (IC 95\%) }\end{array}$ \\
\hline Primaria & $\begin{array}{l}\text { Continuada } \\
\text { Suspendida }\end{array}$ & $\begin{array}{l}26 \\
33\end{array}$ & $\begin{array}{c}1 \\
1,28(1,22 \text { a } 1,34)\end{array}$ \\
\hline Secundaria & $\begin{array}{l}\text { Continuada } \\
\text { Suspendida }\end{array}$ & $\begin{array}{l}54 \\
82\end{array}$ & $\begin{array}{c}1 \\
1,46(1,41 \text { a } 1,51)\end{array}$ \\
\hline Total & $\begin{array}{l}\text { Continuada } \\
\text { Suspendida }\end{array}$ & $\begin{array}{l}41 \\
54\end{array}$ & $\begin{array}{c}1 \\
1,37(1,34 \text { a } 1,41)\end{array}$ \\
\hline
\end{tabular}

CV: cardiovasculares; IC: Intervalo de confianza.

\section{Conclusión}

La discontinuación del consumo a largo plazo de aspirina a bajas dosis se asoció con un aumento del riesgo de eventos $\mathrm{CV}$ de más del $30 \%$, que comenzó tan pronto como los pacientes la suspendieron y no disminuyó con el tiempo. Este riesgo fue mayor en pacientes con antecedentes $\mathrm{CV}$.

Fuente de financiamiento/ conflicto de interés de los autores: Este estudio fue financiado en parte por laboratorios AstraZeneca, (análisis estadístico).

\section{Comentario}

Impulsada por la gran reducción del $50 \%$ de riesgo relativo de muerte o infarto de miocardio observado en los primeros ensayos controlados aleatorizados (ECA) de pacientes con síndrome coronario agudo, la aspirina ha desempeñado un papel fundamental en el tratamiento y la prevención de la enfermedad CV durante más de 30 años ${ }^{1,2}$. Si bien el presente estudio es de gran envergadura e incluye a toda la población sueca que toma aspirina con gran calidad de registro, existen consideraciones importantes para tomar en cuenta al momento de evaluar los resultados. Las estimaciones de riesgo observadas fueron ligeramente más pronunciadas que las encontradas en el meta-análisis de datos de participantes individuales de $\mathrm{ECA}^{3}$. Una posible explicación para las estimaciones más altas podría ser un cierto grado de confusión residual a pesar de los ajustes multivariables para la edad, sexo, ciertas comorbilidades y drogas. De hecho, no se consideran factores de riesgo CV conocidos como el sedentarismo, la hipercolesterolemia o el tabaquismo. Muchos de estos factores pueden asociarse con un mayor riesgo de eventos CV, ya sea en forma directa o mediante una adherencia subóptima a la aspirina y otros medicamentos preventivos. Los factores asociados con la falta de adherencia intencional también incluyen la preferencia individual del paciente, la decisión del médico, la información demográfica del paciente y las contraindicaciones (ya sean reales o percibidas), y la mayoría de estas variables pudieron ser explicadas ${ }^{4}$. Si bien existe consenso en que se debe administrar aspirina a pacientes con enfermedad CV conocida, existen controversias sobre su uso en prevención primaria, debido al balance poco claro entre los beneficios preventivos y el riesgo de sangrado ${ }^{5-7}$. Por el contrario, el grupo de trabajo de servicios preventivos de EE.UU. (USPSTF, por sus siglas en inglés) recomienda el uso de aspirina en dosis bajas para la prevención primaria de la enfermedad CV y del cáncer colorrectal en adultos de 50 a 59 años de edad que tienen un riesgo de enfermedad CV a 10 años de al menos $10 \%$ y estén dispuestos a tomar esta droga diariamente durante al menos 10 años. La decisión puede ser individualizada en personas entre 60 y 69 años de edad, y no hay evidencia suficiente por fuera de este rango etario ${ }^{8}$. A pesar de que al menos cuatro estudios de aspirina para prevención primaria están en curso $^{7}$, la pregunta específica de si la monoterapia a largo plazo con esta droga puede interrumpirse con seguridad podría no responderse con ECA, y este estudio sueco ilustra el valor de los registros a gran escala. Especialmente en prevención secundaria, descontinuar la aspirina a largo plazo fuera del contexto de una cirugía mayor o sangrado parece riesgoso, y se deben hacer esfuerzos para mejorar la adherencia ${ }^{9}$.

Conclusiones del comentador

No puede afirmarse si la discontinuación de aspirina es el factor causal de aumento de eventos. Este estudio podría considerarse en el contexto de toda la evidencia disponible a la hora de tomar decisiones compartidas. 
Agustina Maturi [ Servicio De Medicina Familiar y Comunitaria, Hospital Italiano de Buenos Aires. agustina.maturi@hospitalitaliano.org.ar ]

Maturi A. La discontinuación de dosis bajas de aspirina se asoció a una mayor incidencia de eventos cardiovasculares. Evid Actual Pract Ambul. 2018;21(4):116-117. Comentado de: Sundström J y col. Low-Dose Aspirin Discontinuation and Risk of Cardiovascular Events: A Swedish Nationwide, Population-Based Cohort Study. Circulation. 2017;136(13):1183-92. PMID: 28947478.

\section{Referencias}

1. Lewis HD Jr y col. Protective effects of aspirin against acute myocardial infarction and death in men with unstable angina. Results of a Veterans Administration Cooperative Study. N Engl J Med 1983;309:396-403.

2. Randomised trial of intravenous streptokinase, oral aspirin, both, or neither among 17,187 cases of suspected acute myocardial infarction: ISIS-2. ISIS-2 (Second International Study of Infarct Survival) Collaborative Group. Lancet 1988;2:349-60.

3. Antithrombotic Trialists' (ATT) Collaboration, Baigent C, Blackwell L, et al. Aspirin in the primary and secondary prevention of vascular disease: collaborative meta-analysis of individual participant data from randomised trials. Lancet 2009;373:1849-60.

4. Pareek M y col. Cardiovascular events after discontinuation of low-dose aspirin. J Thorac Dis. 2018;10(1):75-78.

5. Amsterdam EA y col. 2014 AHA/ACC Guideline for the Management of Patients with Non-ST-Elevation Acute Coronary Syndromes: a report of the American College of Cardiology/ American Heart Association Task Force on Practice Guidelines. J Am Coll Cardiol 2014;64:e139-228. 10.1016/j.jacc.2014.09.017

6. Ibanez B y col. 2017 ESC Guidelines for the management of acute myocardial infarction in patients presenting with ST-segment elevation: The Task Force for the management of acute myocardial infarction in patients presenting with ST-segment elevation of the European Society of Cardiology (ESC). Eur Heart J 2018;39:119-77.

7. Piepoli MF y col. 2016 European Guidelines on cardiovascular disease prevention in clinical practice: The Sixth Joint Task Force of the European Society of Cardiology and Other Societies on Cardiovascular Disease Prevention in Clinical Practice (constituted by representatives of 10 societies and by invited experts)Developed with the special contribution of the European Association for Cardiovascular Prevention \& Rehabilitation (EACPR). Eur Heart J 2016;37:2315-81.

the European Association for Cardiovascular Prevention \& Rehabilitation (EACPR). Eur Heart J 2016;37:2315-81. 8. Bibbins-Domingo K, U.S. Preventive Services Task Force Aspirin Use

9. Kolandaivelu K y col. Non-adherence to cardiovascular medications. Eur Heart J 2014;35:3267-76

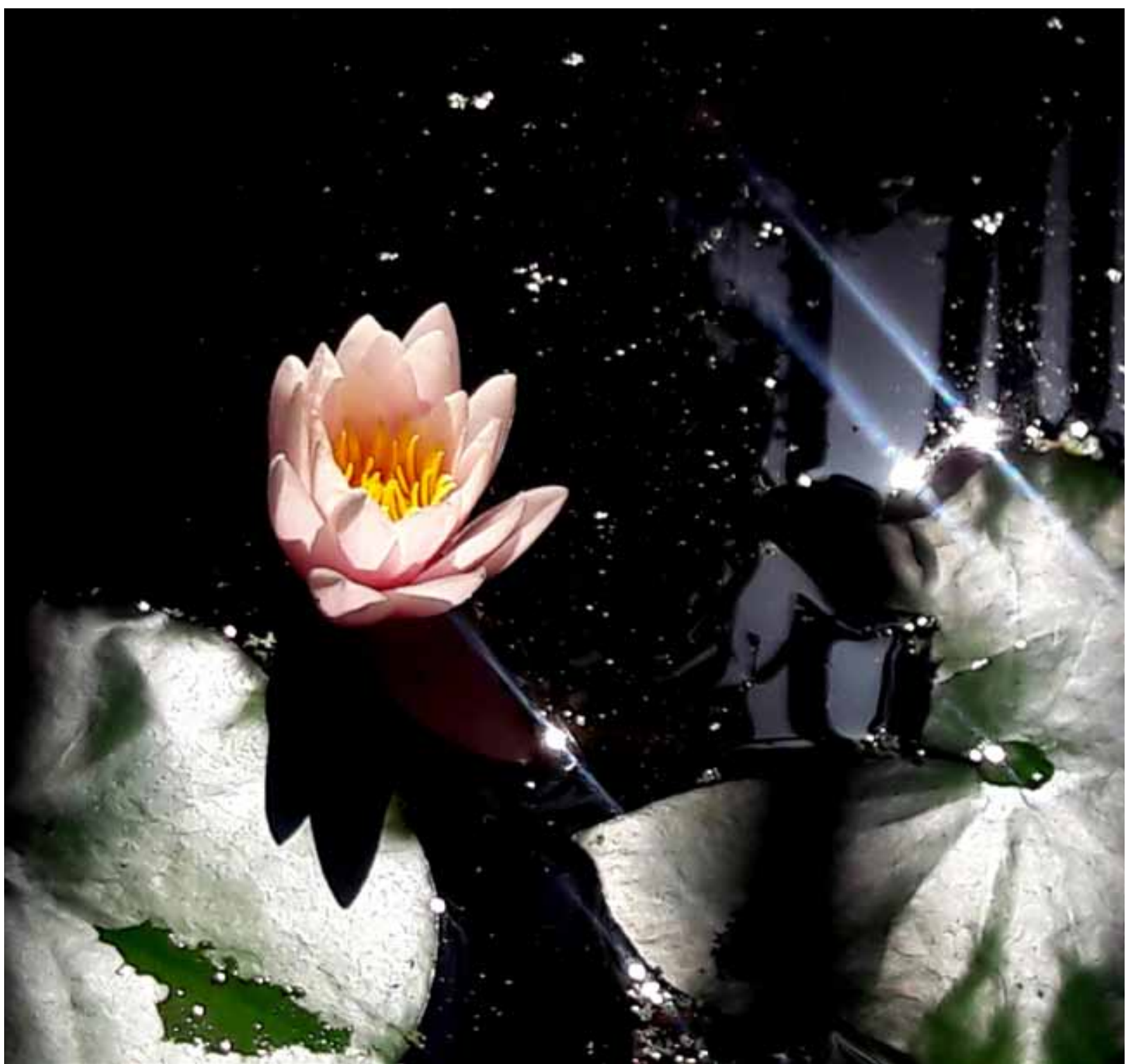

Cortesía de Silvina Spina. 